\title{
Prognostic value of the pretreatment systemic immune-inflammation index (SII) in patients with non-small cell lung cancer: a meta-analysis
}

\author{
Yan Wang ${ }^{1 \#}$, Yina $\mathrm{Li}^{2 \#}$, Pingrun Chen ${ }^{2}$, Wenying $\mathrm{Xu}^{2}$, Yanming $\mathrm{Wu}^{1}$, Guowei Che ${ }^{1}$ \\ ${ }^{1}$ Department of Thoracic Surgery, West China Hospital, Sichuan University, Chengdu 610041, China; ${ }^{2}$ West China School of Medicine, Sichuan \\ University, Chengdu 610041, China \\ Contributions: (I) Conception and design: G Che; (II) Administrative support: G Che; (III) Provision of study materials or patients: Y Wang, Y Li; (IV) \\ Collection and assembly of data: Y Wang, Y Li; (V) Data analysis and interpretation: P Chen, W Xu, Y Wu; (VI) Manuscript writing: All authors; (VII) \\ Final approval of manuscript: All authors. \\ \#These authors contributed equally to this work. \\ Correspondence to: Guowei Che. Department of Thoracic Surgery, West China Hospital, Sichuan University, Guoxuexiang No. 37, Chengdu 610041, \\ China. Email: cheguowei_hx@aliyun.com.
}

\begin{abstract}
Background: The objective of this study is to explore the association between the pretreatment systemic immune-inflammation index (SII) and prognosis in non-small cell lung cancer (NSCLC) patients.

Methods: A systemic literature search of PubMed, EMBASE, the Web of Science, the Cochrane Library, China National Knowledge Infrastructure (CNKI), Wanfang, VIP and SinoMed databases was performed from January 1, 1966 to April 15, 2019, to identify potential studies that assessed the prognostic role of the pretreatment SII in NSCLC. The hazard ratio (HR) and $95 \%$ confidence interval (CI) were combined to evaluate the correlation of the pretreatment SII with overall survival (OS), disease-free survival (DFS), progression-free survival (PFS) and cancer-specific survival (CSS) in NSCLC patients.

Results: A total of 9 studies involving 2,441 patients were eventually included. An elevated pretreatment SII indicated significantly poorer OS ( $\mathrm{HR}=1.88,95 \% \mathrm{CI}: 1.50-2.36 ; \mathrm{P}<0.001)$ with high heterogeneity $\left(\mathrm{I}^{2}=60.6 \%, \mathrm{P}=0.019\right)$, DFS/PFS ( $\left.\mathrm{HR}=2.50,95 \% \mathrm{CI}: 1.20-5.20 ; \mathrm{P}=0.014\right)$ with high heterogeneity $\left(\mathrm{I}^{2}=58.2 \%\right.$, $\mathrm{P}=0.092)$ and $\mathrm{CSS}(\mathrm{HR}=1.852$, 95\% CI: 1.185-2.915; $\mathrm{P}=0.007)$. Subgroup analyses further verified the above results. In addition, compared with the neutrophil to lymphocyte ratio (NLR) and the platelet to lymphocyte ratio (PLR), the SII showed a much higher prognostic value in NSCLC.

Conclusions: The pretreatment SII may serve as a useful prognostic indicator in NSCLC and contribute to prognosis evaluation and treatment strategy formulation. However, more well-designed studies are warranted to verify our findings.
\end{abstract}

Keywords: Systemic immune-inflammation index (SII); non-small cell lung cancer (NSCLC); prognosis; meta-analysis

Submitted May 21, 2019. Accepted for publication Aug 08, 2019.

doi: 10.21037/atm.2019.08.116

View this article at: http://dx.doi.org/10.21037/atm.2019.08.116

\section{Introduction}

Lung cancer is the most common tumor globally and is characterized by insidious early symptoms, rapid progression and a poor prognosis (1). Non-small cell lung cancer (NSCLC) accounts for approximately $85 \%$ of all lung cancer cases (2). In 2016, the number of patients who died from lung cancer increased to 1.7 million, making it the third leading cause of death worldwide $(2,3)$. Despite developments in the early diagnosis and treatment of lung cancer, the prognosis remains poor due to local recurrence or distal metastases (4).

Clinical studies have demonstrated that the inflammatory response plays an important role in tumor progression, 
invasion and metastasis by upregulating inflammation to accelerate tumor angiogenesis and reduce anticancer activities (5). In recent years, inflammatory biomarkers, such as $\mathrm{C}$-reactive protein (CRP), the platelet to lymphocyte ratio (PLR), the neutrophil to lymphocyte ratio (NLR), and the monocyte to lymphocyte ratio (MLR), have been proven to be correlated with cancer prognosis (6). The systemic immune-inflammation index (SII) is a new inflammatory biomarker and is defined as the platelet count $\times$ neutrophil count/lymphocyte count (7). According to previous studies, the SII may have high prognostic value in cancer patients (8). However, there still exists no consensus among the pretreatment SII and survival of NSCLC patients. Therefore, we performed the current meta-analysis to determine the prognostic value of the pretreatment SII in NSCLC.

\section{Methods}

\section{Search strategy}

Relevant studies were searched through PubMed, EMBASE, the Web of Science, the Cochrane Library, China National Knowledge Infrastructure (CNKI), Wanfang, VIP and SinoMed databases from the date of establishment until April 15, 2019. The following terms were used: "lung", "pulmonary", "tumor", "cancer", "carcinoma", "neoplasm", "systemic immune-inflammation index" and "SII", and the search strategy used both MeSH terms and free-test words to increase the sensitivity. Furthermore, the references listed in the included studies were also evaluated.

\section{Inclusion and exclusion criteria}

Inclusion criteria were as follows: (I) articles assessing the relation between the pretreatment SII and the survival of patients diagnosed with NSCLC pathologically; (II) neutrophil, platelet and lymphocyte counts were measured before any treatments, including neoadjuvant chemoradiotherapy, chemoradiotherapy, surgery and targeted therapy; (III) no clinical or laboratory evidence of infection, hematological or autoimmune diseases; (IV) no use of anti-inflammatory or immunosuppressive drugs; (V) the SII was defined as the neutrophil count $\times$ platelet count/ lymphocyte count; (VI) the outcomes of interest included overall survival (OS), cancer-specific survival (CSS), diseasefree survival (DFS) or progression-free survival (PFS) with hazard ratios (HRs) and the corresponding $95 \%$ confidence intervals (95\% CIs); and (8) Newcastle-Ottawa quality assessment scale (NOS) $\geq 6$ (9).

Exclusion criteria were as follows: (I) letters, metaanalyses, editorials, expert opinions, case reports and reviews; (II) nonhuman studies; and (III) if the data were duplicated or overlapped, only the latest study was included.

\section{Study selection}

All searched results were evaluated according to the Preferred Reporting Items for Systematic Reviews and Meta-analyses (PRISMA) statement (10). First, titles and abstracts were screened to identify related studies. Then, full tests were reviewed carefully. The study selection was completed by two independent investigators (Yan Wang and Yina Li).

\section{Data extraction and quality assessment}

Two authors (Yan Wang and Yina Li) extracted data independently, and any disagreement was resolved by discussion until a consensus was reached. The following information was collected: the name of the first author, year of publication, study period, country, sample size, sex tumor-node-metastasis (TNM) stage, treatment, followup period, SII cut-off, outcome, source of HR and HR with $95 \%$ CI. Data were collected by using an excel sheet (Microsoft Corporation).

Quality assessment of the included studies was performed according to the NOS by two independent researchers (Yan Wang and Yina Li) (9). Studies with a score of 6 or higher were defined as high-quality studies.

\section{Statistical analysis}

All analyses were conducted with STATA (version 12.0; Stata Corporation). HRs and $95 \%$ CIs were combined to evaluate the association of the pretreatment SII with prognosis in patients with NSCLC. They were either directly extracted from each study whenever available estimated from Kaplan-Meier (K-M) curves according to the methods reported by Tierney et al. (11). The Higgins $\mathrm{I}^{2}$ statistic and Cochran's Q test were used to evaluate heterogeneity among studies. Significant heterogeneity was defined as $\mathrm{P}<0.10$ and $/$ or $\mathrm{I}^{2}>50 \%$, and when significant heterogeneity was observed, the random-effects model was used; otherwise, the fixed-effects model was used (12). Begg's funnel plot and Egger's linear regression test were 


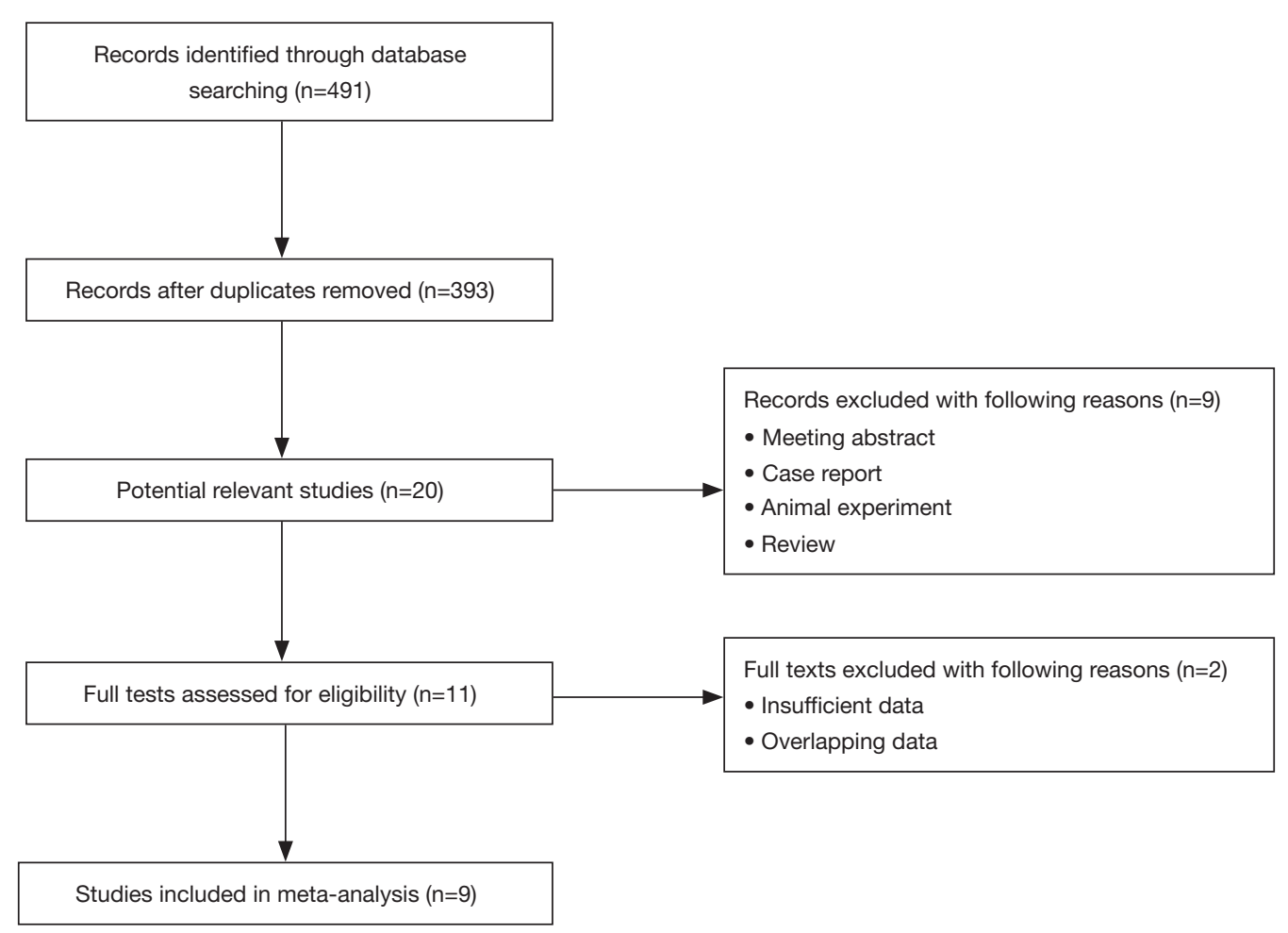

Figure 1 Flow diagram of the literature review.

performed to evaluate publication bias, and significant publication bias was defined as $\mathrm{P}<0.05$ (13).

\section{Results}

\section{Characteristics of the included studies}

The flow diagram is presented in Figure 1. The electronic search yielded 491 studies. After removing duplicates and screening titles and abstracts, 11 studies were assessed by full-tests for eligibility. Finally, a total of 9 articles involving 2,441 patients were included (14-22).

The included studies were all retrospective and published during 2017-2019, with a sample size between 30 and 569 . One of the 9 studies was conducted in Japan (18), and the others were conducted in China (14-17,19-22). The SII cut-off values ranged from 395.4 to 1,218.81. More detailed information is shown in Table 1.

\section{Meta and subgroup analysis results}

A total of 7 studies involving 2,070 patients reported the association of the pretreatment SII with OS in NSCLC patients. The results showed that a high pretreatment SII indicated poor OS ( $\mathrm{HR}=1.88,95 \% \mathrm{CI}: 1.50-2.36$; $\mathrm{P}<0.001)$ with high heterogeneity $\left(\mathrm{I}^{2}=60.6 \%, \mathrm{P}=0.019\right)$ (Figure 2). Subgroup analyses based on the TNM stage and treatment further supported the above results and demonstrated that the TNM stage and treatment method were both potential causes of the significant heterogeneity (Table 2).

Three studies involving 351 patients reported the correlation of the pretreatment SII with DFS/PFS. The combined HR was 2.50 (95\% CI: $1.20-5.20 ; \mathrm{P}=0.014$ ) with high heterogeneity $\left(\mathrm{I}^{2}=58.2 \%, \mathrm{P}=0.092\right)$, which indicated that an elevated pretreatment SII was a negative predictor of DFS/PFS in NSCLC patients (Figure 3) (Table 2).

Only one study reported the relation between the pretreatment SII and CSS in NSCLC patients and indicated that patients with a high pretreatment SII were more likely to have worse CSS (HR =1.852, 95\% CI: 1.185-2.915; $\mathrm{P}=0.007)($ Table 2).

\section{Comparison of the prognostic values of the SII with the NLR and PLR}

Several studies have also reported an association of the NLR and PLR with OS or DFS/PFS. After comparing the 


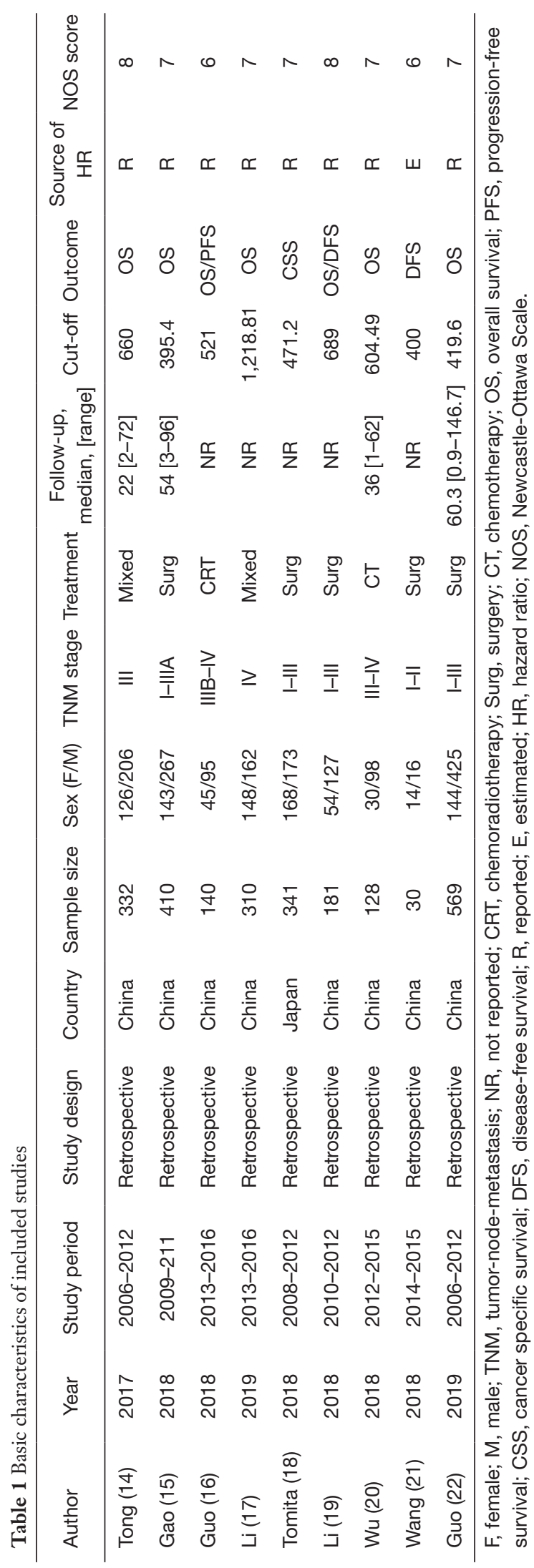

SII with the NLR and PLR in NSCLC patients, only the SII was significantly correlated with OS (HR $=1.82 ; 95 \%$ CI: $1.41-2.35 ; \mathrm{P}<0.001)$ and DFS/PFS (HR $=1.96 ; 95 \% \mathrm{CI}$ : $1.36-2.90 ; \mathrm{P}<0.001)$ in NSCLC patients, which indicated that the SII was superior to the NLR and PLR in predicting the prognosis of NSCLC patients (Table 3).

\section{Sensitivity analysis}

Due to the significant heterogeneity among the included studies, a sensitivity analysis was performed, which showed that the pooled results were still stable after excluding any single study (Figure 4).

\section{Publication bias}

Begg's funnel plot $(\mathrm{P}>0.999)$ (Figure 5) was symmetric, and the $\mathrm{P}$ value of Egger's test was 0.636 , which both indicated no significant publication bias.

\section{Discussion}

It is well known that the inflammatory response has a close relationship with cancer (23). In recent years, we have found that inflammatory infiltration plays an important role in the development of cancers. In NSCLC, many inflammatory cells, including tumor-associated macrophages, tumorinfiltrating lymphocytes, tumor-associated neutrophils and T, B, and NK cells, compose the tumor stroma (24). These cells also contribute to the enlargement and metastasis of cancer tissue through the cytokines they secrete (25). For example, CXCR2 and CXCL8 can promote the processes of angiogenesis, tumor growth and cell proliferation (26). Due to the convenience, low cost and rapidity of the detection of systemic inflammatory markers, studies on inflammatory biomarkers of cancer prognosis are increasing. There are two types of inflammatory markers: the first type is derived from CRP and ALB, and the second type is derived from leukocyte-related inflammation indexes, such as the PLR, NLR, and SII. The latter have been proven to be clinically important in many types of cancer, such as lung cancer, hepatic carcinoma, gastrointestinal cancer and colorectal cancer (27-30). The SII, defined as platelet $\times$ neutrophil/ lymphocyte, is a combination of the PLR and NLR and has been demonstrated to show high prognostic value in some malignant solid tumors (31-34).

By reviewing studies about the SII and the prognosis of NSCLC patients, we found that a higher SII indicated 


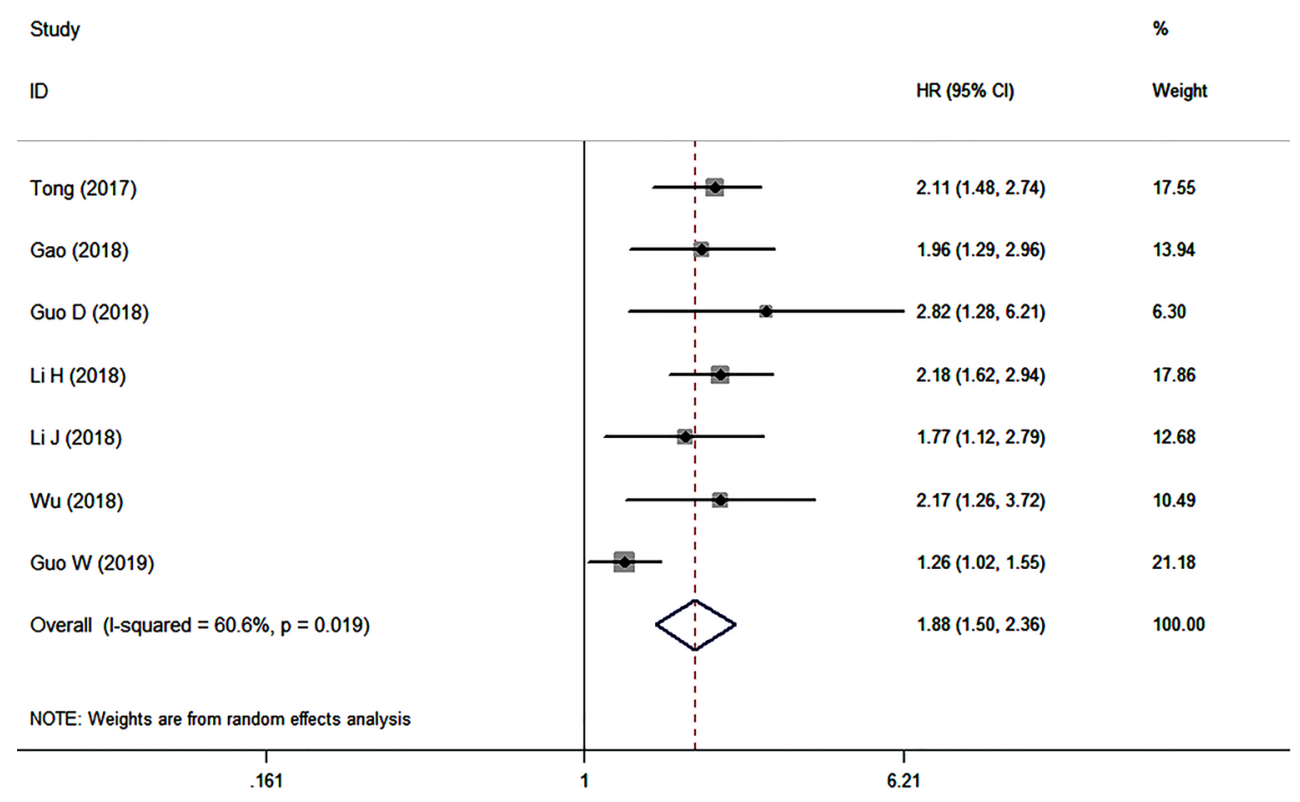

Figure 2 Forest plot of the association between pretreatment SII and overall survival. SII, systemic immune-inflammation index.

Table 2 Meta and subgroup analyses

\begin{tabular}{|c|c|c|c|c|c|}
\hline Analysis & No. of studies & $\mathrm{HR}(95 \% \mathrm{Cl})$ & $P$ values for $\mathrm{HR}$ & $\mathrm{I}^{2}(\%)$ & $\mathrm{P}$ value for heterogeneity \\
\hline \multicolumn{6}{|l|}{ TNM stage } \\
\hline Advanced stage & 4 & $2.18(1.80-2.65)$ & $<0.001$ & 0.0 & 0.926 \\
\hline Mixed & 3 & $1.55(1.15-2.11)$ & 0.004 & 55.8 & 0.104 \\
\hline \multicolumn{6}{|l|}{ Treatment } \\
\hline Mixed & 2 & $2.14(1.73-2.65)$ & $<0.001$ & 0.0 & 0.875 \\
\hline Surgery & 3 & $1.55(1.15-2.11)$ & 0.004 & 55.8 & 0.104 \\
\hline Chemoradiotherapy & 2 & $2.36(1.51-3.68)$ & $<0.001$ & 0.0 & 0.587 \\
\hline $\begin{array}{l}\text { Disease-free survival/ } \\
\text { progression-free survival }\end{array}$ & 3 & $2.50(1.20-5.20)$ & 0.014 & 58.2 & 0.092 \\
\hline
\end{tabular}

TNM, tumor-node-metastasis; HR, hazard ratio; $\mathrm{Cl}$, confidence interval.

a worse prognosis. Several hypotheses may contribute to this consequence. Neutrophils secrete cytokines and chemokines, such as vascular endothelial growth factor (VEGF), to enhance tumor angiogenesis, promote circulating tumor cell adhesion and facilitate distant metastasis (35). Platelets can prevent circulating tumor cells from immune attack and help circulating tumor cells metastasize via blood transmission (36). Moreover, lymphocytes are involved in the acquired immune system, which is indispensable in the body immune defense and immune surveillance (37). Based on these mechanisms, a higher SII combined with increased counts of neutrophils or platelets or a decreased count of lymphocytes leads to enhanced tumor angiogenesis, adhesion, metastasis and poor immune clearance of cancer cells. Therefore, an elevated pretreatment SII is associated with poor outcomes 


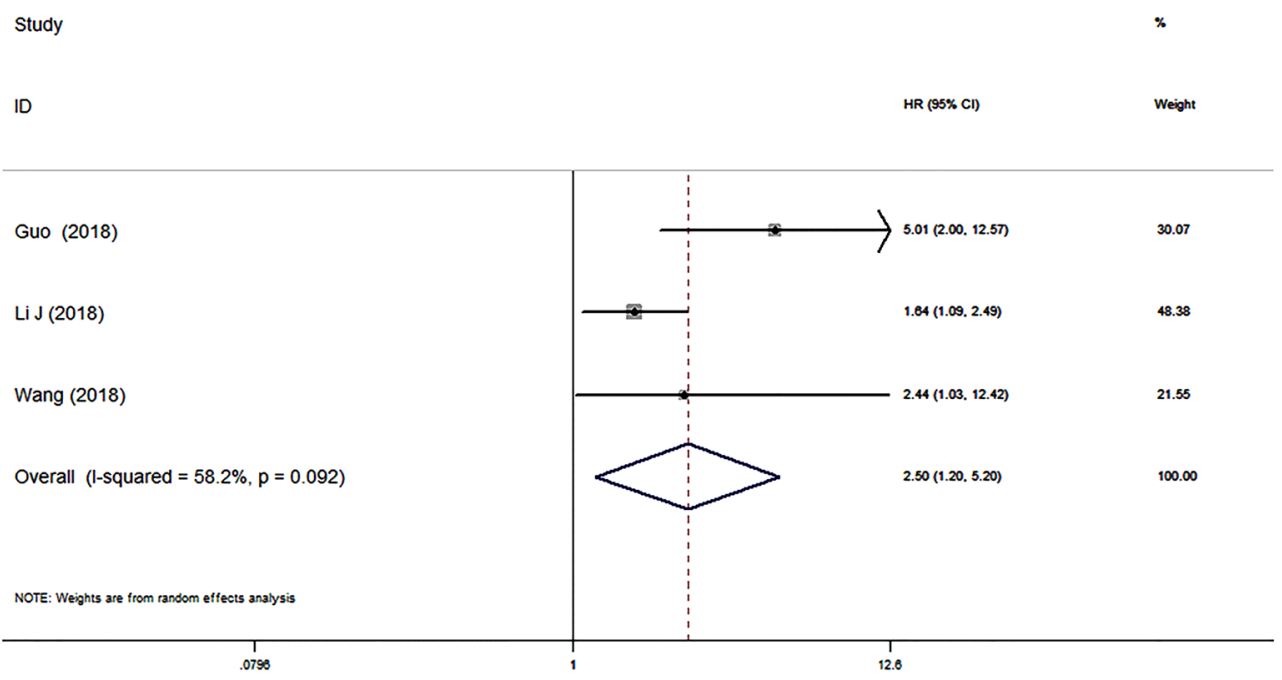

Figure 3 Forest plot of the association between pretreatment SII and disease-free survival/progression-free survival. SII, systemic immuneinflammation index.

Table 3 Comparison of prognostic values of SII with NLR and PLR

\begin{tabular}{|c|c|c|c|c|c|c|c|}
\hline Variables & No. of studies & \multicolumn{2}{|c|}{ SII } & \multicolumn{2}{|c|}{ NLR } & \multicolumn{2}{|c|}{ PLR } \\
\hline OS & 6 & $1.82(1.41-2.35)$ & $<0.001$ & $1.15(0.83-1.59)$ & 0.395 & $1.16(1.01-1.34)$ & 0.042 \\
\hline DFS/PFS & 2 & $1.96(1.36-2.90)$ & $<0.001$ & $1.02(0.71-1.47)$ & 0.916 & $1.01(0.66-1.55)$ & 0.951 \\
\hline
\end{tabular}

SII, systemic immune inflammation index; NLR, neutrophil to lymphocyte ratio; PLR, platelet to lymphocyte ratio; HR, hazard ratio; Cl, confidence interval; OS, overall survival; DFS, disease-free survival; PFS, progression-free survival.

in cancer patients.

For patients with severe systemic immune inflammation, some immune checkpoint inhibitors (ICIs), such as nivolumab, pembrolizumab and atezolizumab, are strongly recommended for use, especially in advanced stage or metastatic patients $(38,39)$. Previous studies have proved that ICIs in first-line or second-line treatment could improve the prognosis of advanced NSCLC patients compared with chemotherapy alone (40). Moreover, Mezquita et al. demonstrated the prognostic value of inflammatory indexes in ICI-treated patients. They introduced a novel index, the lung immune prognostic index (LIPI), which is based on a lactate dehydrogenase (LDH) level greater than the upper limit of normal and a derived neutrophil/(leukocyte minus neutrophil) (dNLR) ratio greater than 3, and reported that the pretreatment LIPI could serve as a useful indicator for predicting OS and PFS in NSCLC patients who were treated with ICIs (41). Unfortunately, no study has explored the prognostic significance of the SII in NSCLC patients who have received ICI therapy until now, which deserves further investigation in the future. Furthermore, it is also necessary to determine whether ICI treatment is beneficial for early-stage NSCLC patients.

The important clinical significance of the current research is that the pretreatment SII could not only predict the prognostic risks of NSCLC patients but also help develop treatment strategies. In detail, compared with patients with a low pretreatment SII, patients with a high pretreatment SII may be recommended for ICI therapy. However, more research is still needed to further determine the indications of ICI treatment for NSCLC patients.

Our study has certain shortcomings. First, only 9 retrospective articles involving 2,441 patients were included, which may cause bias due to the restricted sample size. Second, all included studies were from China or Japan; therefore, the prognostic value of the SII in NSCLC 


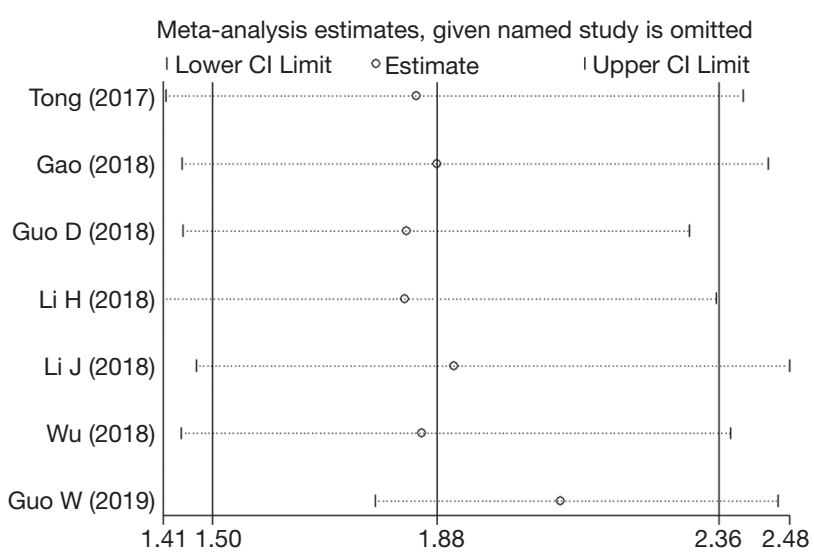

Figure 4 Sensitivity analysis of the association between pretreatment SII and overall survival. SII, systemic immuneinflammation index.

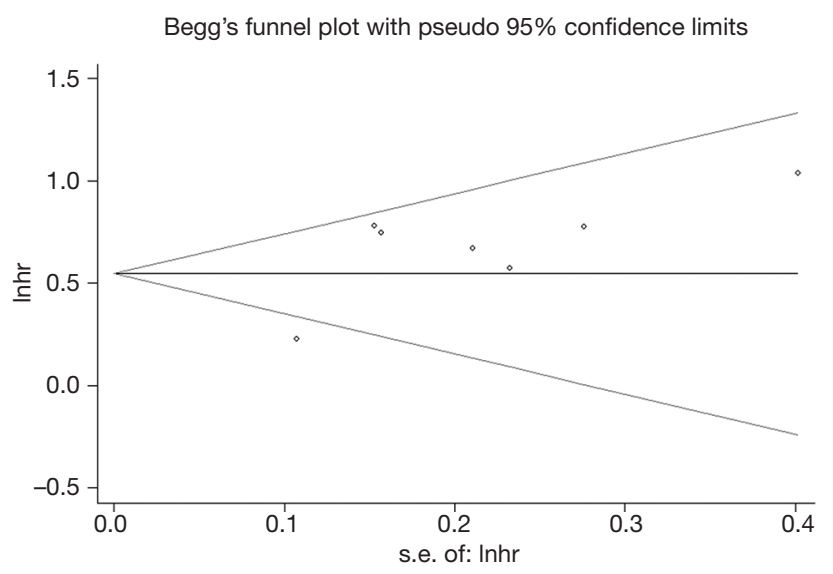

Figure 5 Begg's funnel plot of the association between pretreatment SII and overall survival. SII, systemic immuneinflammation index.

patients from other countries or regions remains unclear. Third, due to the lack of original data, we were unable to conduct more subgroup analyses based on other factors, such as sex, age, and comorbidities.

In conclusion, the pretreatment SII may serve as a useful prognostic indicator in NSCLC and contribute to prognosis evaluation and treatment strategy formulation. However, more prospective and well-designed studies are warranted to verify our findings.

\section{Acknowledgments}

None.

\section{Footnote}

Conflicts of Interest: The authors have no conflicts of interest to declare.

Ethical Statement: All procedures performed in studies that involved human participants were in accordance with the ethical standards of the institutional and/or national research committee and with the 1964 Helsinki Declaration and its later amendments or comparable ethical standards. For this type of study, formal consent was not required. The authors are accountable for all aspects of the work in ensuring that questions related to the accuracy or integrity of any part of the work are appropriately investigated and resolved.

\section{References}

1. Siegel RL, Miller KD, Jemal A. Cancer statistics, 2015. CA Cancer J Clin 2015;65:5-29.

2. Dela Cruz CS, Tanoue LT, Matthay RA. Lung cancer: Epidemiology, etiology, and prevention. Clin Chest Med 2011;32:605-44.

3. Jemal A, Bray F, Center MM, et al. Global cancer statistics. CA Cancer J Clin 2011;61:69-90.

4. Yu Y, Qian L, Cui J. Value of neutrophil-to-lymphocyte ratio for predicting lung cancer prognosis: A meta-analysis of 7,219 patients. Mol Clin Oncol 2017;7:498-506.

5. Candido J, Hagemann T. Cancer-related inflammation. J Clin Immunol 2013;33 Suppl 1:S79-84.

6. Yang R, Chang Q, Meng X, et al. Prognostic value of Systemic immune-inflammation index in cancer: A metaanalysis. J Cancer 2018;9:3295-302.

7. Zhong JH, Huang DH, Chen ZY. Prognostic role of systemic immune-inflammation index in solid tumors: a systematic review and meta-analysis. Oncotarget 2017;8:75381-8.

8. Geng Y, Shao Y, Zhu D, et al. Systemic ImmuneInflammation Index Predicts Prognosis of Patients with Esophageal Squamous Cell Carcinoma: A Propensity Score-matched Analysis. Sci Rep 2016;6:39482.

9. Stang A. Critical evaluation of the Newcastle-Ottawa scale for the assessment of the quality of nonrandomized studies in meta-analyses. Eur J Epidemiol 2010;25:603-5.

10. Moher D, Liberati A, Tetzlaff J, et al. Preferred reporting items for systematic reviews and meta-analyses: the PRISMA statement. Int J Surg 2010;8:336-41.

11. Tierney JF, Stewart LA, Ghersi D, et al. Practical methods 
for incorporating summary time-to-event data into metaanalysis. Trials 2007;8:16.

12. Barili F, Parolari A, Kappetein PA, et al. Statistical Primer: heterogeneity, random- or fixed-effects model analyses? Interact Cardiovasc Thorac Surg 2018;27:317-21.

13. Herrmann D, Sinnett P, Holmes J, et al. Statistical controversies in clinical research: publication bias evaluations are not routinely conducted in clinical oncology systematic reviews. Ann Oncol 2017;28:931-7.

14. Tong YS, Tan J, Zhou XL, et al. Systemic immuneinflammation index predicting chemoradiation resistance and poor outcome in patients with stage III non-small cell lung cancer. J Transl Med 2017;15:221.

15. Gao Y, Zhang H, Li Y, et al. Preoperative increased systemic immune-inflammation index predicts poor prognosis in patients with operable non-small cell lung cancer. Clin Chim Acta 2018;484:272-7.

16. Guo D, Zhang J, Jing W, et al. Prognostic value of systemic immune-inflammation index in patients with advanced non-small-cell lung cancer. Future Oncol 2018;14:2643-50.

17. Li H, Wang G, Zhang H, et al. Prognostic role of the systemic immune-inflammation index in brain metastases from lung adenocarcinoma with different EGFR mutations. Genes Immun 2019;20:455-61.

18. Tomita M, Ayabe T, Maeda R, et al. Comparison of Inflammation-Based Prognostic Scores in Patients undergoing Curative Resection for Non-small Cell Lung Cancer. World J Oncol 2018;9:85-90.

19. Li J. Analysis of cancer complex systemic inflammation score and the prognosis of non-small lung cancer. Shandong: Medical College of Shandong University, 2018.

20. Wu L. The correlation between inflammatory composite index and prognosis in advanced non-small cell lung cancer. Zunyi: Zunyi Medical College, 2018.

21. Wang B, Yao Y, Wang J, et al. Significance of inflammatory index in patients before radical operation for early nonsmall cell lung cancer. Modern Oncol 2018;26:1198-202.

22. Guo W, Cai S, Zhang F, et al. Systemic immuneinflammation index (SII) is useful to predict survival outcomes in patients with surgically resected non-small cell lung cancer. Thorac Cancer 2019;10:761-8.

23. Dolan RD, McSorley ST, Horgan PG, et al. The role of the systemic inflammatory response in predicting outcomes in patients with advanced inoperable cancer: Systematic review and meta-analysis. Crit Rev Oncol Hematol 2017;116:134-46.

24. Mierke CT, Sauer F, Grosser S, et al. The two faces of enhanced stroma: Stroma acts as a tumor promoter and a steric obstacle. NMR Biomed 2018;31:e3831.

25. Atretkhany KN, Drutskaya MS, Nedospasov SA, et al. Chemokines, cytokines and exosomes help tumors to shape inflammatory microenvironment. Pharmacol Ther 2016;168:98-112.

26. Rivas-Fuentes S, Salgado-Aguayo A, Pertuz Belloso S, et al. Role of Chemokines in Non-Small Cell Lung Cancer: Angiogenesis and Inflammation. J Cancer 2015;6:938-52.

27. Suner A, Carr BI, Akkiz H, et al. Inflammatory markers $\mathrm{C}$-reactive protein and PLR in relation to HCC characteristics. J Transl Sci 2019. doi: 10.15761/ JTS.1000260.

28. Zhang $\mathrm{Y}$, Lin S, Yang $\mathrm{X}$, et al. Prognostic value of pretreatment systemic immune-inflammation index in patients with gastrointestinal cancers. J Cell Physiol 2019;234:5555-63.

29. Passardi A, Scarpi E, Cavanna L, et al. Inflammatory indexes as predictors of prognosis and bevacizumab efficacy in patients with metastatic colorectal cancer. Oncotarget 2016;7:33210-9.

30. Zhou ZQ, Pang S, Yu XC, et al. Predictive Values of Postoperative and Dynamic Changes of Inflammation Indexes in Survival of Patients with Resected Colorectal Cancer. Curr Med Sci 2018;38:798-808.

31. Chen JH, Zhai ET, Yuan YJ, et al. Systemic immuneinflammation index for predicting prognosis of colorectal cancer. World J Gastroenterol 2017;23:6261-72.

32. Fu H, Zheng J, Cai J, et al. Systemic ImmuneInflammation Index (SII) is Useful to Predict Survival Outcomes in Patients After Liver Transplantation for Hepatocellular Carcinoma within Hangzhou Criteria. Cell Physiol Biochem 2018;47:293-301.

33. Wang L, Wang C, Wang J, et al. A novel systemic immune-inflammation index predicts survival and quality of life of patients after curative resection for esophageal squamous cell carcinoma. J Cancer Res Clin Oncol 2017;143:2077-86.

34. Hu B, Yang XR, Xu Y, et al. Systemic immuneinflammation index predicts prognosis of patients after curative resection for hepatocellular carcinoma. Clin Cancer Res 2014;20:6212-22.

35. Dvorak HF. Tumor Stroma, Tumor Blood Vessels, and Antiangiogenesis Therapy. Cancer J 2015;21:237-43.

36. Li N. Platelets in cancer metastasis: To help the "villain" to do evil. Int J Cancer 2016;138:2078-87.

37. Mantovani A, Allavena P, Sica A, et al. Cancer-related inflammation. Nature 2008;454:436-44. 
38. Passiglia F, Galvano A, Rizzo S, et al. Looking for the best immune-checkpoint inhibitor in pre-treated NSCLC patients: An indirect comparison between nivolumab, pembrolizumab and atezolizumab. Int J Cancer 2018;142:1277-84.

39. Berghoff AS, Bellosillo B, Caux C, et al. Immune checkpoint inhibitor treatment in patients with oncogeneaddicted non-small cell lung cancer (NSCLC): summary of a multidisciplinary round-table discussion. ESMO Open 2019;4:e000498.

Cite this article as: Wang Y, Li Y, Chen P, Xu W, Wu Y, Che G. Prognostic value of the pretreatment systemic immuneinflammation index (SII) in patients with non-small cell lung cancer: a meta-analysis. Ann Transl Med 2019;7(18):433. doi: 10.21037/atm.2019.08.116
40. Huemer F, Lang D, Westphal T, et al. Baseline Absolute Lymphocyte Count and ECOG Performance Score Are Associated with Survival in Advanced Non-Small Cell Lung Cancer Undergoing PD-1/PD-L1 Blockade. J Clin Med 2019. doi: 10.3390/jcm8071014.

41. Mezquita L, Auclin E, Ferrara R, et al. Association of the Lung Immune Prognostic Index With Immune Checkpoint Inhibitor Outcomes in Patients With Advanced Non-Small Cell Lung Cancer. JAMA Oncol 2018;4:351-7. 